\title{
In Patients With COPD, Treatment With a Combination of Formoterol and Ipratropium Is More Effective Than a Combination of Salbutamol and Ipratropium*
}

\author{
A 3-Week, Randomized, Double-Blind, Within- \\ Patient, Multicenter Study
}

\author{
Anthony D. D'Urzo, MD; Mariá Cristina De Salvo, MD; Alicia Ramirez-Rivera, MD; \\ Joâo Almeida, MD; Lazaros Sichletidis, MD, FCCP; Guenter Rapatz, MSc; and \\ John Kottakis, MD; for the FOR-INT-03 Study Group $f$
}

\begin{abstract}
Study objectives: To compare the efficacy of adding formoterol or salbutamol to regular ipratropium bromide treatment in COPD patients whose conditions were suboptimally controlled with ipratropium bromide alone.

Design: A randomized, double-blind, double-dummy, two-period, crossover clinical trial.

Setting: Twenty-four clinics and university medical centers in nine countries.

Patients: One hundred seventy-two patients with baseline $\mathrm{FEV}_{1} \leq 65 \%$ predicted, with $\mathrm{FEV}_{1}$ reversibility to salbutamol not exceeding the normal variability of the measurement, and symptomatic despite regular treatment with ipratropium bromide.

Interventions: Each patient received two treatments in random order: either inhaled formoterol dry powder, $12 \mu \mathrm{g}$ bid, in addition to ipratropium bromide, $40 \mu \mathrm{g}$ qid for 3 weeks, followed by salbutamol, $200 \mu \mathrm{g}$ qid, in addition to ipratropium, $40 \mu \mathrm{g}$ qid for 3 weeks, or vice versa.

Measurements and results: Efficacy end points included morning premedication peak expiratory flow (PEF) during the last week of treatment (primary end point), the area under the curve (AUC) for FEV $_{1}$ measured for $6 \mathrm{~h}$ after morning dose on the last day of treatment, and symptom scores (from daily diary recordings). Morning PEF and the AUC for $F E V_{1}$ were significantly better for formoterol/ ipratropium than for salbutamol/ipratropium $(p=0.0003$ and $p<0.0001$, respectively). The formoterol/ipratropium combination also induced a greater improvement in mean total symptom scores $(p=0.0042)$. The safety profile of the two treatments was comparable.

Conclusions: In COPD patients requiring combination bronchodilator treatment, the addition of formoterol to regular ipratropium treatment is more effective than the addition of salbutamol.
\end{abstract}

(CHEST 2001; 119:1347-1356)

Key words: $\beta_{2}$-agonist; COPD; formoterol; ipratropium; salbutamol

Abbreviations: $\mathrm{ANOVA}=$ analysis of variance; $\mathrm{AUC}=$ area under the curve; $\mathrm{CI}=$ confidence interval; $\mathrm{ITT}=$ intent to treat; $\mathrm{PEF}=$ peak expiratory flow; QoL = quality of life; SGRQ $=$ St. George’s Respiratory Questionnaire

${ }^{*}$ From Novartis Pharmaceuticals (Dr. Kottakis and Mr. Rapatz), Horsham, UK; the Primary Care Asthma Clinic (Dr. D'Urzo), Toronto, Canada; Hospital General de Agudos "E." Tornu (Dr. De Salvo), Buenos Aires, Argentina; Hospital de Enfermedades Cardiovasculares y del Torax IMSS (Dr. Ramirez-Rivera), Monterrey, Mexico; Hospital Sao Joao, Servico de Pneumologia (Dr. Almeida), Porto, Portugal; and Papanicolaou Hospital, Pneumonological University Clinic (Dr. Sichletidis), Thessaloniki, Greece. $\uparrow$ A complete list of participants is located in the Appendix. This study was supported by a research grant from Novartis Pharma A.G., Basel, Switzerland. According to their statements, the authors and all study investigators have not made any financial arrangement whereby the value of the compensation could be influenced by the outcome of the study; have not received significant payments from the sponsor of other sorts, excluding the costs for conducting the study; do not have a proprietary or financial interest in the test product such as patent, trademark, copyright, or licensing agreements; do not hold a significant equity interest in the sponsor of the study (exceeding $\$ 50,000$ [US]). John Kottakis, MD, and Guenter Rapatz, MSc, hold permanent positions with Novartis Pharmaceuticals.

Manuscript received May 30, 2000; revision accepted November 21, 2000.

Correspondence to: John Kottakis, MD, Novartis HRC, Wimblehurst Rd, Horsham, RH12 5AB, UK; e-mail: ioannis.kottakis@ pharma.novartis.com 
$\mathbf{C}$ OPD has been recognized as a major health problem for approximately 50 years. ${ }^{1}$ Its prevalence and the disease-related morbidity and mortality are still rising in contrast to many leading chronic diseases. ${ }^{2-4}$ In those patients with advanced disease, pharmacologic therapy is used with the aim to reduce symptoms and to improve exercise capacity.

Typically, bronchodilators are prescribed for maintenance therapy of the reversible obstructive component of the disease. ${ }^{5}$ In addition, it has been postulated that the improvements in symptoms observed following bronchodilator therapy are related to reductions in gas trapping and in the amount of dynamic hyperinflation, which in turn result in reductions in the overall work of breathing. ${ }^{6,7}$ This especially holds true for patients with minimally reversible or irreversible obstruction and explains the positive perception these patients have toward bronchodilator therapy.

The bronchodilators most often used belong to the pharmacologic classes of the anticholinergics and short-acting $\beta_{2}$-adrenoceptor agonists. ${ }^{2-4}$ For patients whose conditions are not sufficiently controlled by monotherapy, current international guidelines for the management of COPD recommend combination therapy withan inhaled anticholinergic and a short-acting $\beta_{2}$-adrenoceptor agonist. ${ }^{2-4}$ This approach recognizes that anticholinergics and $\beta_{2}$ adrenoceptor agonists achieve their bronchodilating effects via different mechanisms that may involve different sites within the airways. ${ }^{8}$ A number of studies $^{9-17}$ have indeed demonstrated that the combination of an inhaled anticholinergic and an inhaled $\beta_{2}$-adrenoceptor agonist is more effective than either agent alone in patients with stable COPD.

The new $\beta_{2}$-adrenoceptor agonists formoterol fumarate and salmeterol xinafoate have a longer duration of action than previously available $\beta_{2}$-adrenoceptor agonists, and can be administered twice daily. This feature may promote compliance with treatment and make these agents an attractive alternative in the treatment of stable COPD. However, their positioning in the management of COPD is not clearly defined since sufficient clinical data are not yet available. ${ }^{2-4,18}$

The objective of this study was to obtain clinical information on the use of formoterol in combination with the inhaled anticholinergic ipratropium bromide in patients with COPD manifesting a need for combination bronchodilator treatment. More specifically, this study was designed to determine whether the coadministration of formoterol and ipratropium bromide improves lung function, symptoms, and quality of life (QoL) when compared with the currently recommended coadministration of ipratropium with the short-acting $\beta_{2}$-adrenoceptor agonist salbutamol sulfate.

\section{MATERIALS AND Methods}

\section{Study Design}

This was a randomized, multicenter, double-blind, doubledummy, two-period, crossover clinical trial comparing the clinical benefits of adding either formoterol (Foradil Aerolizer; Novartis; Basel, Switzerland), $12 \mu \mathrm{g}$ bid via dry-powder inhaler, or salbutamol (Ventolin; Glaxo Smith Kline; Uxbridge, UK), $200 \mu \mathrm{g}$ qid via pressurized metered-dose inhaler (100 $\mu \mathrm{g} / \mathrm{puff})$, to ipratropium bromide (Atrovent; Boehringer Ingelheim; Ingelheim, Germany), $40 \mu \mathrm{g}$ qid via pressurized metered-dose inhaler (20 $\mu \mathrm{g} / \mathrm{puff})$, in adult male and female patients with COPD.

The study was carried out in 24 centers in nine countries $(2$ centers in Argentina, 6 in Canada, 2 in Greece, 1 in Italy, 2 in Mexico, 3 in Norway, 2 in Poland, 3 in Portugal and 3 in Spain). It was approved by the appropriate ethics committee at each site and was conducted according to the principles of good clinical practice.

The study had three parts. Part 1 was the run-in period of 2 -weeks' duration that followed screening visit 1 . During this period, patients received regular treatment with ipratropium bromide. Baseline measurements were performed, and the eligibility of screened patients to participate in the randomized treatment periods was assessed.

Part 2 and part 3 constituted the randomized treatment periods. Each period was of 3-weeks' duration. At the end of the run-in period, at visit 2 , eligible patients were randomized to one of the two treatment sequences: (1) formoterol plus placebo salbutamol in addition to ipratropium bromide in part 2 , salbutamol plus placebo formoterol in addition to ipratropium bromide in part 3; and (2) salbutamol plus placebo formoterol in addition to ipratropium bromide in part 2, formoterol plus placebo salbutamol in addition to ipratropium bromide in part 3.

Blinding was achieved by using the double-dummy technique. Since only efficacy parameters from the last 7 days of each treatment period were used for analysis, no washout periods were necessary. Patients returned to the clinic for evaluation at the end of each treatment period (visit 3 and visit 4). Additional inhalations of ipratropium bromide were allowed, as required, throughout the whole study. However, such "rescue" use of ipratropium bromide was restricted to a maximum of eight puffs per day.

\section{Study Patients}

Inclusion criteria were the following: cooperative outpatients with a diagnosis of COPD according to the American Thoracic Society guidelines ${ }^{2}$ who gave written informed consent; age $\geq 40$ years; current or previous smokers ( $>10$ pack-years); prebronchodilator baseline $\mathrm{FEV}_{1} \leq 65 \%$ of the predicted normal value and at least $1.0 \mathrm{~L}$ and $\mathrm{FEV}_{1} \leq 70 \% \mathrm{FVC}$; an increase in $\mathrm{FEV}_{1}$ of at least $5 \%$ from baseline value and $<12 \%$ of predicted normal value $30 \mathrm{~min}$ following the inhalation of salbutamol, $400 \mu \mathrm{g}$; a total symptom score from the daily diary recordings of $>1$ on at least 3 of the last 7 days of the run-in period; and use of ipratropium bromide for at least 1 month prior to the screening visit.

Specific exclusion criteria included the following: current or childhood asthma; respiratory tract infection within 1 month before the screening visit or during the run-in period; hospitalization or emergency department treatment for an acute COPD exacerbation in the month preceding the screening visit or during the run-in period; need for long-term oxygen therapy; QTc $>0.46 \mathrm{~s}$ at the screening visit; and treatment with inhaled corticosteroids or oral xanthines that had been started or discon- 
tinued or subjected to any change in the daily dose or dosing schedule in the month preceding the screening visit or during the run-in period.

\section{Efficacy Parameters}

The primary efficacy variable was mean morning premedication peak expiratory flow (PEF) over the last week of each treatment period (the last 7 days before visit 3 and visit 4 , including the day of the visit).

Secondary efficacy variables were $\mathrm{FEV}_{1}$ and $\mathrm{FVC}$ before the morning dose of study medications at visit 3 and visit 4; the area under the curve (AUC) for $\mathrm{FEV}_{1}$ and $\mathrm{FVC}$ recorded at 5 min, 15 min, $30 \mathrm{~min}, 60 \mathrm{~min}$, and hourly thereafter over the 6-h interval following the morning dose of study medications at visit 3 and visit 4; peak $\mathrm{FEV}_{1}$ after the morning dose of study medications at visits 3 and 4; total symptom scores from the daily diary recordings; QoL; and number of COPD exacerbations.

At visits 1,2, and 3, the patients received a diary to be used until the following visit. The patients were instructed to complete the diary daily in the morning, before the first dose of study medications, considering events over the last $24 \mathrm{~h}$. They recorded their morning PEF and the number of inhalations of rescue medication, and rated each of six questions regarding the symptoms they had experienced on a 4-point scale (from 0 to 3 ). There were six individual scores for impairment of daily activities, breathlessness, severity of respiratory symptoms during night, coughing, amount of sputum, and breathlessness on rising. For each day, the total score was determined. This ranged from 0 (best value) to 18 (worst value).

$\mathrm{PEF}, \mathrm{FEV}_{1}$ and $\mathrm{FVC}$ were measured as previously described. ${ }^{19}$ Patients and investigators were required to perform $\mathrm{PEF}, \mathrm{FEV}_{1}$, and FVC measurements at the same time of the day in order to minimize the normal day-time variability of the measurements. Rescue inhalations of ipratropium bromide were to be avoided within $8 \mathrm{~h}$ before and during spirometric measurements at visits 2,3 , and 4 .

QoL was evaluated at visits 2, 3, and 4 by using a validated instrument, the disease-specific St. George's Respiratory Questionnaire (SGRQ) ${ }^{20}$ However, the time interval in this study was shorter than the minimum 1-month period recommended by the questionnaire's developer (P.W. Jones, $\mathrm{PhD}$; personal communication; September 2000). For patients in non-English-speaking countries, validated translations to local language were used.

Concerning the number of COPD exacerbations, the levels of exacerbations were identified as follows: (1) "bad days," defined as days with at least two symptom items with a score of $\geq 2$ as recorded in the diary or a reduction of PEF from baseline of $>20 \%$; or (2) COPD-related hospitalizations, excluding emergency department visits, that did not require overnight stay.

\section{Safety Variables}

Any and all adverse events were recorded and monitored throughout the study. An adverse event was defined as any symptom, physical sign, syndrome, or disease that occurred after start of treatment with the study drug or was present at the start of treatment with the study drug and worsened. Patients underwent a physical examination at the screening visit and at each subsequent visit. An ECG was performed at the screening visit. $\mathrm{BP}$ and heart rate were recorded before dosing and during the serial spirometric measures performed $>6 \mathrm{~h}$ after the first dose of study medications at visit 3 and visit 4 .

\section{Statistical Analysis}

As the design of the study foresaw testing for superiority, intent-to-treat (ITT) analyses were performed. ${ }^{21}$ Three different
ITT populations were identified. The first population (ITT-1) comprised all randomized patients who received the study medications and from whom at least one efficacy measurement during the run-in period and one efficacy measurement during each treatment period were obtained. For the morning premedication $\mathrm{PEF}$, additional analyses were separately performed in ITT-1 patients who had no COPD exacerbations during the study (ITT-2 population), and in ITT-1 patients who presented with at least one exacerbation (ITT-3 population).

For some variables, such as morning premedication PEF, the mean values over the last week of each treatment period were used. This value was calculated if measurements on at least 5 days were available.

For the primary efficacy end point, mean morning premedication PEF averaged over the last 7 days of each treatment period, an analysis of variance (ANOVA) was performed to test for differences between treatment groups in the ITT-1 population. The dependent variable was the change in PEF from baseline in each treatment period, where baseline value was the mean morning premedication PEF over the last 7 days of the run-in period. The model included treatment, treatment period, treatment sequence, and patient within treatment sequence as effects. A significance level of 0.05 (two sided) was applied.

For all the other efficacy end points, ANOVA was performed as described above, using the corresponding data recorded during the run-in period as baseline values, unless otherwise specified. The AUC for $\mathrm{FEV}_{1}$ measured before and over $6 \mathrm{~h}$ after the morning dose of study medications at visit 3 and visit 4 was calculated using the trapezoidal rule.

On the basis of a previous study in patients with reversible obstructive airway disease and a study in patients with COPD, ${ }^{22}$ the SD for the difference between treatments in the mean morning premedication PEF was estimated to be $40 \mathrm{~L} / \mathrm{min}$. Assuming a treatment difference in favor of formoterol of 10 $\mathrm{L} / \mathrm{min}$, the sample size was calculated to be 130 patients in order to detect this difference with a power of $80 \%$ and a significance level of 0.05 , two sided. Taking into account dropouts and missing data, we planned to randomize a minimum of 160 patients.

\section{RESULTs}

\section{Study Patients}

A total of 252 patients were screened, and 172 patients were randomized to each treatment sequence as reported in Table 1 . Of the randomized patients, 159 completed the study and 13 withdrew prematurely, all during the first treatment period. Four patients were discontinued due to adverse events, and all these discontinuations occurred during treatment with the salbutamol/ipratropium combination. Four patients withdrew their consent, two patients were unavailable for follow-up, two patients violated the protocol, and one patient had an unsatisfactory therapeutic effect on treatment with the formoterol/ipratropium combination. Two of the 13 patients who withdrew prematurely completed the first treatment period (salbutamol/ipratropium combination) but did not start the next treatment period. Of the 163 patients who started the formoterol/ ipratropium treatment period, 159 completed that treatment period. Similarly, 161 of the 168 patients 
Table 1-Patient Disposition by Treatment Sequence and by Treatment*

\begin{tabular}{|c|c|c|}
\hline Variables & FI & SI \\
\hline \multicolumn{3}{|l|}{ By treatment sequencet } \\
\hline Patients randomized & $88(100)$ & $84(100)$ \\
\hline Entered first crossover period & $88(100)$ & $84(100)$ \\
\hline Completed first crossover period & $84(95.5)$ & $77(91.7)$ \\
\hline Entered second crossover period & $84(95.5)$ & $75(89.3)$ \\
\hline Completed second crossover period & $84(95.5)$ & $75(89.3)$ \\
\hline \multicolumn{3}{|l|}{ Discontinued first crossover period } \\
\hline Total & $4(4.5)$ & $7(8.3)$ \\
\hline Adverse events & 0 & $3(3.6)$ \\
\hline Withdrawal of consent & $2(2.3)$ & $2(2.4)$ \\
\hline Unavailable for follow-up & $1(1.1)$ & $1(1.2)$ \\
\hline Unsatisfactory therapeutic effect & $1(1.1)$ & 0 \\
\hline Protocol violation & 0 & $1(1.2)$ \\
\hline \multicolumn{3}{|l|}{ Did not start second crossover period } \\
\hline Total & 0 & $2(2.4)$ \\
\hline Adverse events & 0 & $1(1.2)$ \\
\hline Protocol violation & 0 & $1(1.2)$ \\
\hline \multicolumn{3}{|l|}{$\begin{array}{l}\text { Discontinued second crossover } \\
\text { period }\end{array}$} \\
\hline Total & 0 & 0 \\
\hline \multicolumn{3}{|l|}{ By treatment } \\
\hline Entered period & $163(100)$ & $168(100)$ \\
\hline Completed period & $159(97.5)$ & $161(95.8)$ \\
\hline \multicolumn{3}{|l|}{ Discontinued period } \\
\hline Total & $4(2.5)$ & $7(4.2)$ \\
\hline Adverse events & 0 & $3(1.8)$ \\
\hline Withdrawal of consent & $2(1.2)$ & $2(1.2)$ \\
\hline Unavailable for follow-up & $1(0.6)$ & $1(0.6)$ \\
\hline Unsatisfactory therapeutic effect & $1(0.6)$ & 0 \\
\hline Protocol violation & 0 & $1(0.6)$ \\
\hline \multicolumn{3}{|l|}{ Did not start next period } \\
\hline Total & 0 & $2(1.2)$ \\
\hline Adverse events & 0 & $1(0.6)$ \\
\hline Protocol violation & 0 & $1(0.6)$ \\
\hline
\end{tabular}

*Data are presented as No. (\%); FI = formoterol/ipratropium combination; $\mathrm{SI}=$ salbutamol/ipratropium combination.

$\nmid$ FI followed by SI; SI followed by FI.

who started the salbutamol/ipratropium treatment period completed that treatment period.

A summary of the demographic and baseline data of the ITT-1 population is reported in Table 2 . Their ages ranged from 40 to 91 years. Most of the patients were male and white.

Concomitant medications, in addition to ipratropium bromide, were inhaled corticosteroids in 65 of the randomized patients $(40.9 \%)$, oral theophylline in 15 patients $(9.4 \%)$, and both inhaled corticosteroids and oral theophylline in 17 patients (10.7\%).

For all spirometric variables measured at the screening visit (visit 1), the mean value was within the range defined in the inclusion criteria. The mean morning premedication $\mathrm{FEV}_{1}$ value recorded at visit 2 , before starting the first treatment period, was comparable to the corresponding value recorded at the screening visit.
Table 2-Summary of Demographic and Baseline Data of ITT-1 Population $(n=159)^{*}$

\begin{tabular}{|c|c|}
\hline Variables & Data \\
\hline Age, yr & $65(9.4)$ \\
\hline \multicolumn{2}{|l|}{ Sex, No. (\%) } \\
\hline Male & $128(80.5)$ \\
\hline Female & $31(19.5)$ \\
\hline \multicolumn{2}{|l|}{ Race, No. (\%) } \\
\hline White & $142(89.3)$ \\
\hline Black & $1(0.6)$ \\
\hline Asian/other & $0 / 16(10.1)$ \\
\hline \multicolumn{2}{|l|}{ Concomitant medications, No. (\%) } \\
\hline Inhaled corticosteroids & $65(40.9)$ \\
\hline Oral theophylline & $15(9.4)$ \\
\hline Both & $17(10.7)$ \\
\hline $\mathrm{FEV}_{1}$ before salbutamol, $\mathrm{L}$ & $1.4(0.36)$ \\
\hline $\mathrm{FEV}_{1}$ before salbutamol, $\%$ predicted & $51.3(10.48)$ \\
\hline $\mathrm{FEV}_{1}$ before salbutamol, $\%$ of $\mathrm{FVC}$ & $52.9(9.13)$ \\
\hline$\Delta \mathrm{FEV}_{1}, \%$ of $\mathrm{FEV}_{1}$ before salbutamol & $11.0(6.25)$ \\
\hline$\Delta \mathrm{FEV}_{1}, \%$ of predicted value & $5.5(2.91)$ \\
\hline $\mathrm{FEV}_{1}$ before study drug at visit $2, \mathrm{~L}$ & $1.4(0.40)$ \\
\hline FVC before study drug at visit $2, \mathrm{~L}$ & $2.6(0.71)$ \\
\hline Mean morning premedication $\mathrm{PEF}, \mathrm{L} / \mathrm{min}$ & $259(80.8)$ \\
\hline Total symptom score (diary) & 5.8 \\
\hline No. of inhalations of rescue medication, puffs/d & 1.9 \\
\hline
\end{tabular}

The mean total symptom score from the daily diary recordings was 5.8. The percentage of patients who needed rescue intake of ipratropium ranged from 37.3 to $43.9 \%$ across days 1 to 14 of the run-in period. The mean number of inhalations of rescue medication was 1.9 puffs per day.

\section{Morning Premedication PEF}

The ITT-1 analysis of the primary efficacy variable included only 144 of the 159 patients in the ITT-1 population. Of the patients not included, one patient had no diary, nine patients had $<5$ PEF measurements during the last 7 days of at least one treatment period, and five patients had $<5$ PEF measurements during the last 7 days of the run-in period. The ITT-2 population consisted of 31 patients who did not have any exacerbation of COPD during the treatment periods. An additional analysis was also performed in the ITT-3 population of 113 patients with at least one COPD exacerbation.

On average, the mean morning premedication $\mathrm{PEF}$ increased during both treatment periods, but the increase was notably higher with the formoterol/ ipratropium combination (Table 3). The change in favor of formoterol/ipratropium was evident and statistically significant in the ITT-1 population as well as in the ITT-2 and ITT-3 populations (Table 3).

The time course of the morning premedication 
Table 3-Mean Morning Premedication PEF (Liters per Minute) Over the Last 7 Days of Each Treatment Period: Change From Baseline and Treatment Contrasts*

\begin{tabular}{|c|c|c|c|}
\hline Populations & FI & SI & FI $-\mathrm{SI}$ \\
\hline \multicolumn{4}{|l|}{ ITT-1 $(\mathrm{n}=144)$} \\
\hline \multicolumn{4}{|l|}{ Descriptive results } \\
\hline Mean & 15.1 & 3.0 & 12.1 \\
\hline $\mathrm{SD}$ & 36.1 & 43.1 & 39.3 \\
\hline \multicolumn{4}{|l|}{ ANOVA results } \\
\hline Treatment contrast & Differenceł幸 & 95\% CI†幸 & $\mathrm{p}$ value $+\underset{+}{t}$ \\
\hline $\mathrm{FI}-\mathrm{SI}$ & 12.1 & $5.6-18.6$ & 0.0003 \\
\hline \multicolumn{4}{|l|}{ ITT-2 $(\mathrm{n}=31)$} \\
\hline \multicolumn{4}{|l|}{ Descriptive results } \\
\hline Mean & 27.8 & 18.1 & 9.7 \\
\hline $\mathrm{SD}$ & 27.2 & 25.1 & 19.5 \\
\hline \multicolumn{4}{|l|}{ ANOVA results } \\
\hline Treatment contrast & Difference $\dagger^{+}$ & $95 \% \mathrm{CI}$ 寺 $^{+}$ & $\mathrm{p}$ value $+\frac{t}{+}$ \\
\hline $\mathrm{FI}-\mathrm{SI}$ & 9.5 & $2.8-16.2$ & 0.0073 \\
\hline \multicolumn{4}{|l|}{ ITT-3 $(\mathrm{n}=113)$} \\
\hline \multicolumn{4}{|l|}{ Descriptive results } \\
\hline Mean & 11.6 & -1.2 & 12.8 \\
\hline $\mathrm{SD}$ & 37.6 & 46.1 & 43.2 \\
\hline \multicolumn{4}{|l|}{ ANOVA results } \\
\hline Treatment contrast & Differenceł幸 & $95 \% \mathrm{CI}$ 幸 & $\mathrm{p}$ value $\frac{+}{+}$ \\
\hline $\mathrm{FI}-\mathrm{SI}$ & 12.7 & $4.6-20.8$ & 0.0023 \\
\hline
\end{tabular}

* See Table 1 for expansion of abbreviations not defined in text. $\nmid$ Difference between treatment least squares means.

+ Based on the model: change from baseline $=$ treatment + treat ment sequence + patient (treatment sequence) + period

PEF in the ITT-1 population is displayed by treatment group in Figure 1. In this graph, the estimated mean (plus 95\% confidence intervals [CIs]) PEF values are displayed per day. For the primary analysis, changes from baseline averaged over the last 7 days of treatment were analyzed. Thus the $95 \%$ CIs largely overlap in Figure 1, whereas for the primary analysis the SD was much smaller and therefore the treatment differences were highly significant. The secondary variables described below were analyzed only on the ITT-1 population.

\section{$\mathrm{FEV}_{1}$ and $\mathrm{FVC}$}

Compared to baseline values, premedication $\mathrm{FEV}_{1}$ increased following 3-weeks' treatment with formoterol/ipratropium and decreased following treatment with salbutamol/ipratropium. The estimated treatment difference was $0.116 \mathrm{~L}$ and was highly statistically significant $(\mathrm{p}<0.0001$; Table 4$)$.

Postmedication $\mathrm{FEV}_{1}$ was higher with formoterol/ ipratropium than with salbutamol/ipratropium, with treatment differences that were highly statistically significant at all time points between $0 \mathrm{~h}$ and $6 \mathrm{~h}$ (Table 4).

The 6-h profile of $\mathrm{FEV}_{1}$ is displayed by treatment in Figure 2. It shows that peak postmedication mean value was achieved $2 \mathrm{~h}$ after dosing with formoterol/ ipratropium and $1 \mathrm{~h}$ after dosing with salbutamol/ ipratropium. The peak postmedication $\mathrm{FEV}_{1}$ was significantly higher with formoterol/ipratropium than with salbutamol/ipratropium $(\mathrm{p}<0.0001)$. The profile also indicates that the $\mathrm{AUC}$ of $\mathrm{FEV}_{1}$ for formoterol/ipratropium was much higher than for salbutamol/ipratropium ( $<<0.0001$; Fig 2, Table 4).

To investigate the supplementary short-term effect of the two treatments, we analyzed the difference between all postmedication time points and the premedication $\mathrm{FEV}_{1}$ value (Table 5). Small but significant differences in favor of salbutamol/ipratropium were found $15 \mathrm{~min}$ and $1 \mathrm{~h}$ after dosing, and significant differences in favor of formoterol/ipratropium were found at $4 \mathrm{~h}$ and $5 \mathrm{~h}$ after dosing (Table 5).

FVC was measured at the same time points as $\mathrm{FEV}_{1}$. The results are illustrated in Figure 3. As for

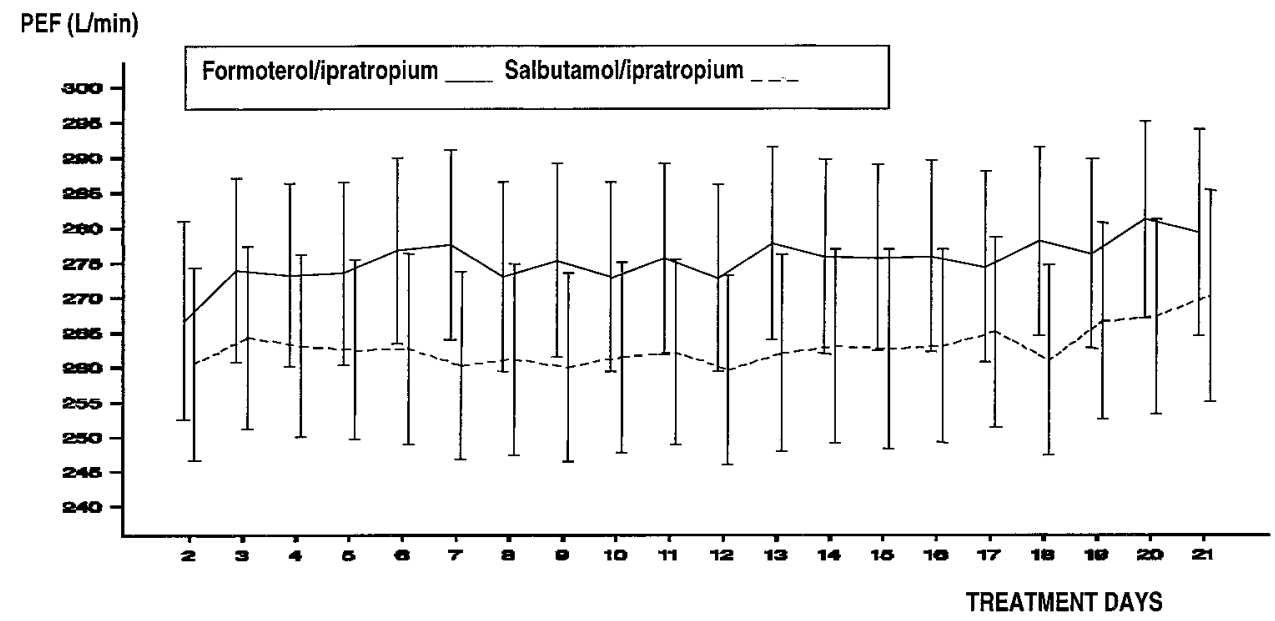

FIGURE 1. Morning premedication PEF during each treatment period. Mean values and 95\% CIs (ITT-1 population). 


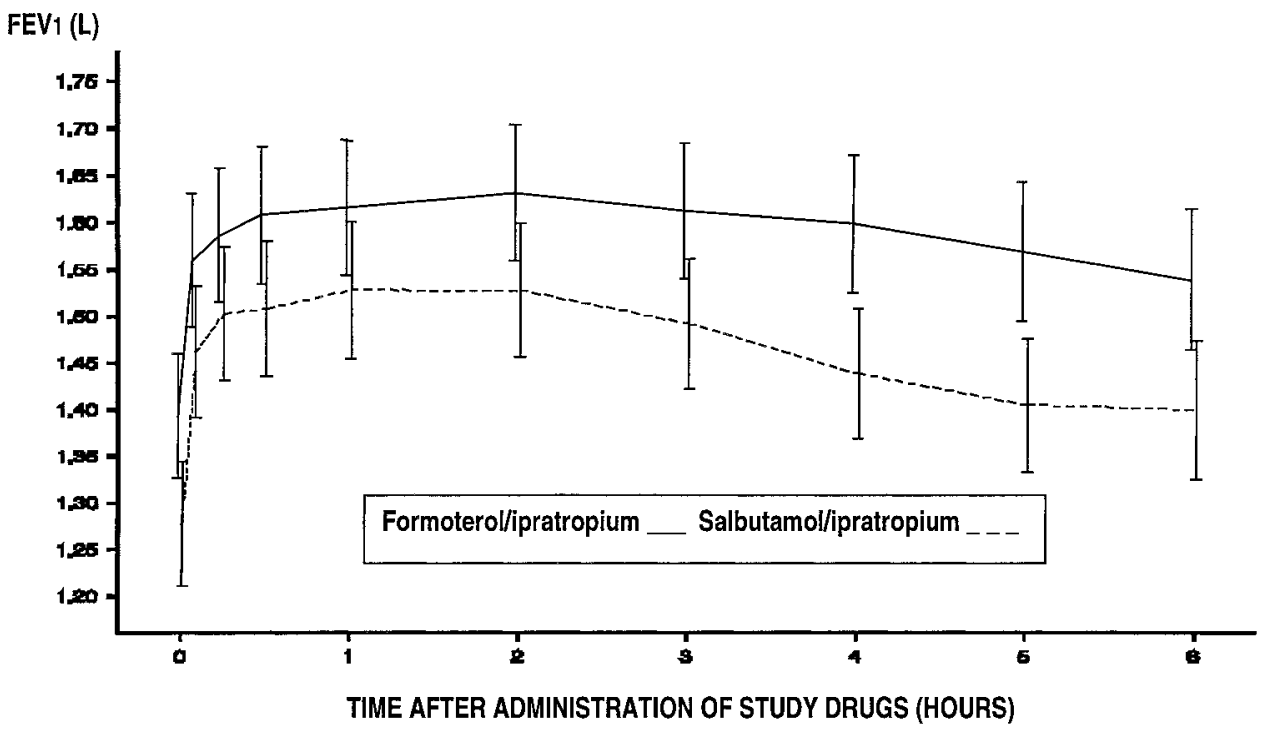

FIGURE 2. Six-hour profile of $\mathrm{FEV}_{1}$ after the morning dose of study medications on the last day of each treatment period. Mean values and $95 \%$ CIs (ITT-1 population).

$\mathrm{FEV}_{1}$ the difference between treatments in $\mathrm{FVC}$ was highly statistically significant at all time points, although slightly less pronounced. The treatment differences between $5 \mathrm{~min}$ and $2 \mathrm{~h}$ postmedication were below the premedication difference and above the premedication difference between $3 \mathrm{~h}$ and $6 \mathrm{~h}$.

\section{Symptom Scores and Inhalation of Rescue Medication}

On average, all mean individual symptom scores were lower with formoterol/ipratropium than with

Table 4-Estimates of Treatment Differences With Associated 95\% CI for FEV (Liters) Measured at Different Time Points After Study Drug Administration at the End of Each Treatment Period in ITT-1 Population*

\begin{tabular}{|c|c|c|c|}
\hline \multirow[b]{2}{*}{ Variables } & \multicolumn{3}{|c|}{$\mathrm{FI}-\mathrm{SI}$} \\
\hline & Difference $+t$ & $95 \% \mathrm{CI}+t+$ & $\mathrm{p}$ Value $+\underset{+}{t}$ \\
\hline Premedication $\mathrm{FEV}_{1}$ & 0.116 & $0.083-0.150$ & $<0.0001$ \\
\hline $\mathrm{FEV}_{1} 5$ min after study drug & 0.097 & $0.062-0.131$ & $<0.0001$ \\
\hline $\mathrm{FEV}_{1} 15$ min after study drug & 0.083 & $0.047-0.119$ & $<0.0001$ \\
\hline $\mathrm{FEV}_{1} 30$ min after study drug & 0.094 & $0.059-0.130$ & $<0.0001$ \\
\hline $\mathrm{FEV}_{1} 1 \mathrm{~h}$ after study drug & 0.087 & $0.049-0.124$ & $<0.0001$ \\
\hline $\mathrm{FEV}_{1} 2 \mathrm{~h}$ after study drug & 0.104 & $0.064-0.143$ & $<0.0001$ \\
\hline $\mathrm{FEV}_{1} 3 \mathrm{~h}$ after study drug & 0.119 & $0.081-0.157$ & $<0.0001$ \\
\hline $\mathrm{FEV}_{1} 4 \mathrm{~h}$ after study drug & 0.159 & $0.120-0.198$ & $<0.0001$ \\
\hline $\mathrm{FEV}_{1} 5 \mathrm{~h}$ after study drug & 0.161 & $0.122-0.199$ & $<0.0001$ \\
\hline $\mathrm{FEV}_{1} 6 \mathrm{~h}$ after study drug & 0.137 & $0.093-0.181$ & $<0.0001$ \\
\hline Peak postmedication $\mathrm{FEV}_{1}$ & 0.105 & $0.067-0.144$ & $<0.0001$ \\
\hline $\mathrm{AUC}$ of $\mathrm{FEV}_{1}$ & 44.5 & $32.3-56.7$ & $<0.0001$ \\
\hline
\end{tabular}

*See Table 1 for expansion of abbreviations not defined in text. $\nmid$ Difference between treatment least squares means.

$\$$ Based on the model: change from baseline $=$ treatment + treatment sequence + patient (treatment sequence) + period. salbutamol/ipratropium, The mean difference between treatments ranged from -0.08 for the amount of sputum to -0.14 for breathlessness on rising and -0.16 for breathlessness during the past $24 \mathrm{~h}$.

The mean total symptom score was 0.6 points lower with formoterol/ipratropium than with salbutamol/ipratropium. This difference was statistically significant $(95 \% \mathrm{CI},-1.01$ to $-0.19 ; \mathrm{p}=0.0042$ ).

The mean number of inhalations of rescue medication during the last 7 days of each treatment period was 1.3 with formoterol/ipratropium and 1.5 with salbutamol/ipratropium. The mean percentage of days with the number of inhalations of rescue medication equal to 0,1 to 2,3 to 4 , and $>4$ was $72.3 \%$, $7.4 \%, 8.0 \%$, and $12.4 \%$ with formoterol/ipratropium and $68.8 \%, 10.1 \%, 8.9 \%$, and $12.2 \%$ with salbutamol/ ipratropium, respectively.

\section{QoL}

At the end of the run-in period and of each treatment period, patients assessed their QoL during the past 3 weeks by means of the SGRQ. Total scores and the scores for each "activity" and "impacts" domain were calculated and analyzed by ANOVA. The results of this analysis are reported in Table 6.

\section{COPD Exacerbations}

The number of patients with no COPD exacerbations during the treatment periods was slightly higher with formoterol/ipratropium than with salbutamol/ipratropium: 55 patients (34.6\%) and 49 patients (30.8\%), 


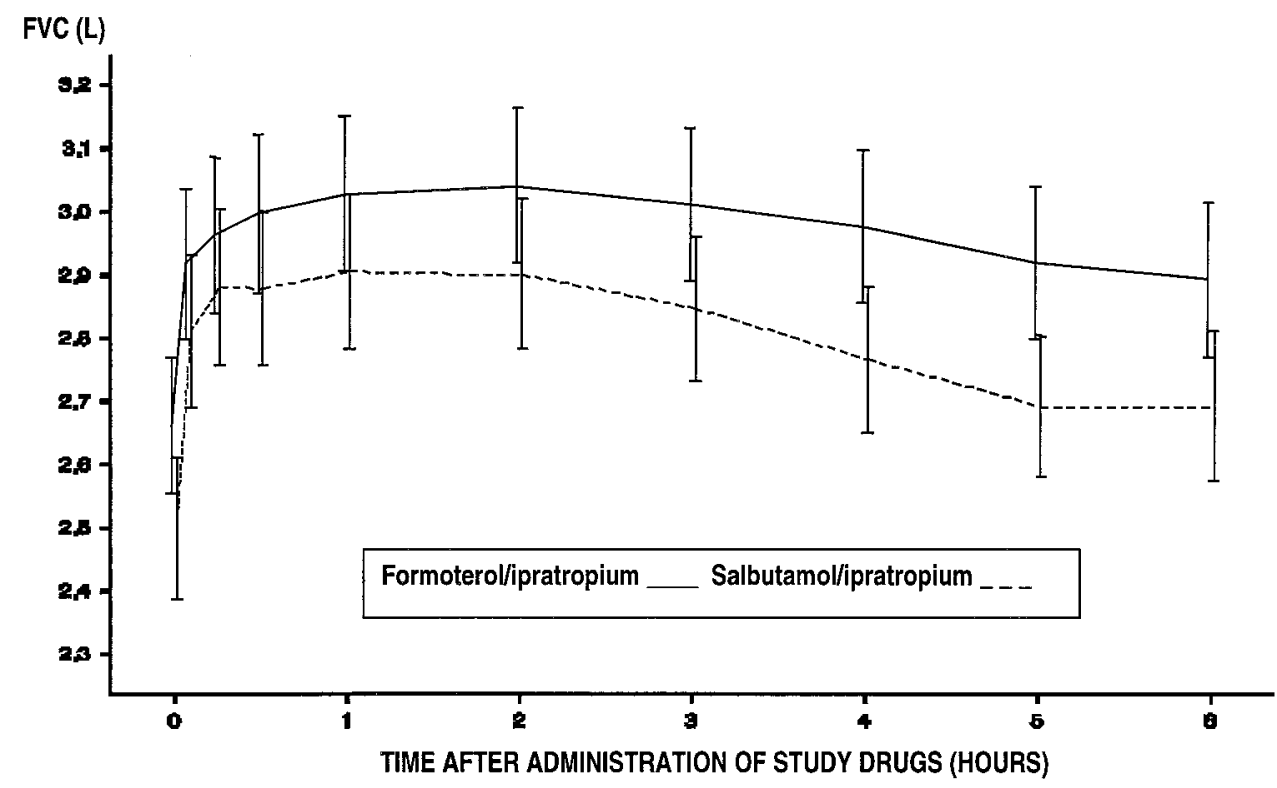

FIGURE 3. Six-hour profile of FVC after the morning dose of study medications on the last day of each treatment period. Mean values and 95\% CIs (ITT-1 population).

respectively. The number of patients with bad days during treatment with formoterol/ipratropium and salbutamol/ipratropium was 104 patients (65\%) and 110 patients $(69 \%)$, respectively. Only one patient was hospitalized during the study because of an exacerbation of COPD, and this event occurred during treatment with salbutamol/ipratropium.

\section{Safety Variables}

No patient died during this study. One patient experienced a serious adverse exacerbation of ob-

Table 5-Estimates of Treatment Differences With Associated $95 \%$ CI for $\triangle F E V_{1}$ (Liters) Measured at Different Time Points After Study Drug Administration at the End of Each Treatment Period in (ITT-1 Population)*

\begin{tabular}{|c|c|c|c|}
\hline \multirow[b]{2}{*}{ Variables } & \multicolumn{3}{|c|}{$\mathrm{FI}-\mathrm{SI}$} \\
\hline & Difference ++ & 95\% CI ${ }^{\dagger}+$ & p Value + \\
\hline Peak postmedication $\Delta \mathrm{FEV}_{1}$ & -0.010 & $-0.041-0.021$ & 0.5092 \\
\hline$\Delta \mathrm{FEV}_{1} 5$ min after study drug & -0.019 & $-0.044-0.007$ & 0.1466 \\
\hline$\Delta \mathrm{FEV}_{1} 15$ min after study drug & -0.036 & $-0.064-0.008$ & 0.0130 \\
\hline$\Delta \mathrm{FEV}_{1} 30 \mathrm{~min}$ after study drug & -0.025 & $-0.051-0.000$ & 0.0545 \\
\hline$\Delta \mathrm{FEV}_{1} 1 \mathrm{~h}$ after study drug & -0.031 & $-0.058-0.003$ & 0.0313 \\
\hline$\Delta \mathrm{FEV}_{1} 2 \mathrm{~h}$ after study drug & -0.012 & $-0.044-0.020$ & 0.4559 \\
\hline$\Delta \mathrm{FEV}_{1} 3 \mathrm{~h}$ after study drug & 0.004 & $-0.030-0.037$ & 0.8315 \\
\hline$\Delta \mathrm{FEV}_{1} 4 \mathrm{~h}$ after study drug & 0.042 & $0.010-0.073$ & 0.0096 \\
\hline$\Delta \mathrm{FEV}_{1} 5 \mathrm{~h}$ after study drug & 0.043 & $0.008-0.078$ & 0.0169 \\
\hline$\Delta \mathrm{FEV}_{1} 6 \mathrm{~h}$ after study drug & 0.018 & $-0.021-0.056$ & 0.3631 \\
\hline AUC of $\Delta \mathrm{FEV}_{1}$ & 1.9 & $-7.4-11.2$ & 0.6892 \\
\hline
\end{tabular}

*See Table 1 for expansion of abbreviations not defined in text. $\nmid$ Difference between treatment least squares means.

+ Based on the model: $\Delta \mathrm{FEV}_{1}=$ treatment + treatment sequence + patient (treatment sequence) + period. structive airways disease associated with an aggravation of diabetes mellitus during treatment with the salbutamol/ipratropium combination and was hospitalized. One patient had a serious adverse event (congestive heart failure) after the end of the study. Three patients were withdrawn during treatment with salbutamol/ipratropium because of nonserious adverse events; one patient, who had a nonserious adverse event during treatment with salbutamol/ ipratropium, did not start the next treatment period because of that event.

Sixteen patients (9.8\%) had 19 adverse events with treatment with formoterol/ipratropium, and 22 patients $(13.1 \%)$ had 34 adverse events with treatment with salbutamol/ipratropium. Six patients (3.7\%) reported respiratory system disorders with formoterol/ ipratropium and 14 patients (8.3\%) with salbutamol/ ipratropium. The adverse events most frequently reported in this body system and overall were dyspnea, exacerbation of obstructive airway disease, and pharyngitis, all occurring more frequently during treatment with the salbutamol/ipratropium combination (five vs two patients, five vs zero patients, and three vs one patients as compared to the formoterol/ ipratropium combination). Of these most frequently reported adverse events, a total of five events were of mild severity (one event of dyspnea and one event of pharyngitis with the formoterol/ipratropium combination, and one event of dyspnea and two events of pharyngitis with the salbutamol/ipratropium combination). Dyspnea was reported three times as being of moderate severity, exacerbation of obstructive airways disease four times, and pharyngitis one time, all 
during treatment with the salbutamol/ipratropium combination. Dyspnea was reported as severe once with both drug combinations and, finally, exacerbation of obstructive airways disease was reported once as being severe with the salbutamol/ipratropium combination. In total, only 1 of the 19 adverse events that occurred with formoterol/ipratropium were severe and 4 events were moderate. In contrast, 4 of the 34 adverse events that occurred with salbutamol/ipratropium were severe and 15 events were moderate.

During treatment with formoterol/ipratropium, three adverse events in three patients were suspected to be drug related. This relationship was suspected for 10 adverse events that occurred in seven patients while receiving treatment with salbutamol/ipratropium. The drug-related adverse events were hypertension, dry mouth, and leg cramps with formoterol/ipratropium, and pharyngitis, dyspnea, dizziness, tremor, leg cramps, coughing, and exacerbation of obstructive airway disease with salbutamol/ ipratropium. There were no relevant changes in vital signs from baseline, and the difference between treatments was very small for all parameters.

\section{Discussion}

The results of this randomized, double-blind, crossover study indicate that the addition of formoterol, $12 \mu \mathrm{g}$ bid, to treatment with ipratropium bromide, $40 \mu \mathrm{g}$ qid, is more effective than the addition of salbutamol, $200 \mu \mathrm{g}$ qid, in patients with COPD who require combined bronchodilator therapy.

Mean morning premedication PEF increased significantly more during treatment with formoterol/ ipratropium than during treatment with salbutamol/ ipratropium. The change from baseline in the ITT-1 population, which included patients with and without COPD exacerbations during the two treatment periods, was $15.1 \mathrm{~L} / \mathrm{min}$ with formoterol/ipratropium and only $3.0 \mathrm{~L} / \mathrm{min}$ with salbutamol/ipratropium.

Table 6-Estimates of Treatment Differences With Associated 95\% CI for the SGRQ Scores in ITT-1 Population After 3 Weeks of Treatment

\begin{tabular}{|c|c|c|c|}
\hline \multirow[b]{2}{*}{ SGRQ Score } & \multicolumn{3}{|c|}{$\mathrm{FI}-\mathrm{SI}$} \\
\hline & Differencełt & 95\% CI†幸 & p Value $+\frac{t}{+}$ \\
\hline Symptoms score & -2.64 & $-5.17--0.11$ & 0.0408 \\
\hline Activity score & -0.64 & $-3.07-1.79$ & 0.6042 \\
\hline Impacts score & -1.52 & $-3.55-0.51$ & 0.1420 \\
\hline Total score & -1.52 & $-3.18-0.14$ & 0.0731 \\
\hline
\end{tabular}

* See Table 1 for expansion of abbreviations not defined in text. $\uparrow$ Difference between treatment least squares means.

${ }_{+}^{+}$Based on the model: change from baseline $=$treatment + treatment sequence + patient (treatment sequence) + period.
This indicates a more sustained improvement in lung function by formoterol and is in keeping with the longer duration of action of this agent as compared to salbutamol.

When considering only patients who had no acute exacerbation of COPD (ITT-2 population), the change in mean morning premedication $\mathrm{PEF}$ was $27.8 \mathrm{~L} / \mathrm{min}$ with formoterol/ipratropium and 18.1 $\mathrm{L} / \mathrm{min}$ with salbutamol/ipratropium. The difference between treatments was still statistically significant and in favor of the formoterol/ipratropium combination, but was smaller than that observed in the ITT-1 population. However, the difference between formoterol/ipratropium and salbutamol/ipratropium became more pronounced when we considered only patients who had at least one COPD exacerbation (ITT-3 population), the changes in PEF being 11.5 $\mathrm{L} / \mathrm{min}$ with formoterol/ipratropium and $-1.2 \mathrm{~L} / \mathrm{min}$ with salbutamol/ipratropium.

The different magnitude of the effect of formoterol and salbutamol in the two subpopulations of patients may be attributed to differences in relative potency and efficacy of the two bronchodilators. ${ }^{23}$ Interestingly, a loss of bronchodilating effectiveness similar to that observed with the salbutamol-containing drug combination in the ITT-3 population has been previously reported with short-acting $\beta_{2}$-adrenoceptor agonists in asthmatic patients during exacerbations associated with viral infections, ${ }^{24-26}$ and has been attributed to a possible abnormality of $\beta_{2}$-adrenoceptor function. ${ }^{27-30}$ To our knowledge, such an observation has not been previously reported in patients with COPD, and we believe that, as an original finding, it warrants further investigation.

The results observed with the mean morning premedication PEF were confirmed by the results of the analysis of mean premedication $\mathrm{FEV}_{1}$ values at the end of each treatment period. These were much better with formoterol/ipratropium than with salbutamol/ipratropium, with a difference between treatments being highly statistically significant.

During the serial measurements of $\mathrm{FEV}_{1}$ following the morning dose of study medication on the last day of each treatment period, postmedication $\mathrm{FEV}_{1}$ was significantly better with formoterol/ipratropium than with salbutamol/ipratropium at any time point during the $6 \mathrm{~h}$. Peak postmedication $\mathrm{FEV}_{1}$ was also significantly higher after the administration of formoterol and ipratropium than after the administration of salbutamol and ipratropium. The difference in bronchodilating potency measured in terms of AUC of $\mathrm{FEV}_{1}$ was in favor of the formoterol/ ipratropium combination and was highly statistically significant.

When considering the changes in $\mathrm{FEV}_{1}$ from the predose value during the $6 \mathrm{~h}$ following the morning 
dose of study medication, the salbutamol/ipratropium combination was better than the formoterol/ ipratropium combination at $5 \mathrm{~min}, 15 \mathrm{~min}, 1 \mathrm{~h}$, and $2 \mathrm{~h}$ after dosing, with statistically significant difference at $15 \mathrm{~min}$ and $1 \mathrm{~h}$. This finding can be at least in part explained by the difference in premedication $\mathrm{FEV}_{1}$ between treatments. Since the mean premedication $\mathrm{FEV}_{1}$ was significantly lower with salbutamol/ ipratropium than with formoterol/ipratropium, as a result of the more prolonged bronchodilating effect of the previous dose of formoterol, there was more room for improvement when the patients received the salbutamol/ipratropium combination. Although the results of a previous study in a small number of COPD patients have indicated that the onset of action of salbutamol may be faster than that of long-acting $\beta_{2}$-adrenoceptor agonists, ${ }^{31}$ other studies on the time course of the effect of formoterol in patients with reversible obstructive airway disease ${ }^{32}$ and COPD $^{33,34}$ do not support this possibility.

The combination of formoterol and ipratropium also provided a superior and sustained symptom relief as compared to the combination of salbutamol and ipratropium. There were appreciable differences between treatments in the individual symptom scores from the patient diary, including the amount of sputum, breathlessness on rising, and breathlessness during the past $24 \mathrm{~h}$. The total symptom scores were significantly lower with formoterol/ipratropium than with salbutamol/ipratropium, and this was associated with a less frequent use of rescue medication for symptom relief during treatment with formoterol and ipratropium. In addition, patients showed a lower number of bad days, with objective and subjective evidence of disease deterioration, on treatment with the combination of formoterol and ipratropium than on treatment with salbutamol and ipratropium. The only hospitalization due to COPD exacerbation occurred during treatment with salbutamol/ipratropium.

Although the treatment periods were limited to 3 weeks, it was possible to detect a difference between the two treatments also in terms of quality of life. All scores of the SGRQ domains and the "total" score were lower with formoterol/ipratropium than with salbutamol/ipratropium, suggesting a possible greater impact on quality of life with the formoterol/ipratropium combination. This beneficial effect due to the addition of formoterol was statistically significant for the score, referring to respiratory symptoms, their frequency and severity. ${ }^{20}$ However, it should be noted that the 3 -week treatment period used is believed to be too short to allow for a full manifestation of the beneficial effects of the tested treatments on QoL (P.W. Jones, PhD; personal communication; Sep- tember 2000), and consequently the QoL results obtained in this study should be interpreted with caution.

The safety profile of the formoterol/ipratropium combination was slightly better than the salbutamol/ ipratropium combination, since the total number of adverse events, the number of drug-related adverse events, and the number of premature discontinuations due to adverse events tended to increase during treatment with salbutamol and ipratropium.

Our results with the combination of salbutamol and ipratropium are similar or better than those reported in patients with COPD treated for 85 days with a marketed combination of the two agents. ${ }^{15}$ This validates our study design and indirectly supports the therapeutic implications of our findings, the most important of which is that formoterol may represent the most effective option for combined treatment with ipratropium bromide when monotherapy with one bronchodilator is not sufficient to provide an optimal control of COPD-related symptoms.

\section{APPENDIX}

Participants: J. Almeida, MD, Porto, Portugal; S. Boucher, MD, Quebec, Canada; J. Castillo, MD, Sevilla, Spain; S. Centanni, MD, Milano, Italy; J. Cordoso, MD, Lisbon, Portugal; M. C. De Salvo, MD, Buenos Aires, Argentina; C. G. Di Bartolo, MD, Buenos Aires, Argentina; A. D'Urzo, MD, Toronto, Canada; A. Eivindson, MD, Arendal, Norway; D. Gorecka, MD, Warsaw, Poland; C. Gratziou, MD, Athens, Greece; J. Kottakis, MD, Horsham, UK; J. Kozielski, MD, Zabrze, Poland; G. Krammer, MSc, Basel, Switzerland; K. E. Langaker, MD, Oslo, Norway; M-H Lindor, MD, Montreal, Canada; R. Maleki-Yazdi, MD, Toronto, Canada; J. R. Manzano, MD, Badalona, Spain; P. Patel, MD, Missisauga, Canada; A. M. Perez, MD, Sabadell, Spain; P. Pfister, MD, Basel, Switzerland; A. Ramirez-Rivera, MD, Monterrey, Mexico City, Mexico; G. Rapatz, MSc, Basel, Switzerland; K. Rimmer, MD, Calgary, Canada; N. Ringdal, MD, Molde, Norway; M. A. Salazar, MD, Mexico; E. Barbeta Sanchez, MD, Granollers, Spain; J. Moutinho dos Santos, MD, Coimbra, Portugal; L. Sichletidis, MD, Thessaloniki, Greece.

ACKNOWLEDGMENT: We are grateful to Professor Paul W. Jones for the QoL results.

\section{REFERENCES}

1 Senior RM, Anthonisen NR. Chronic obstructive pulmonary disease (COPD). Am J Respir Crit Care Med 1998; 157:139147

2 American Thoracic Society. Standards for the diagnosis and care of patients with chronic obstructive pulmonary disease. Am J Respir Crit Care Med 1995; 152:77-120

3 Siafakas NM, Vermeire P, Pride NB, et al. Optimal assessment and management of chronic obstructive pulmonary disease (COPD). Eur Respir J 1995; 8:1398-1420

4 British Thoracic Society. BTS guidelines for the management of chronic obstructive pulmonary disease. Thorax 1997; 52(suppl 5):S1-S28

5 Friedman M. Changing practices in COPD: a new pharmacologic treatment algorithm. Chest 1995; 107(suppl):S194S197 
6 O’Donnell DE, Webb KA. Exertional breathlessness in patients with chronic airflow limitation: the role of lung hyperinflation. Am Rev Respir Dis 1993; 148:1351-1357

7 Calverley P, Bellamy D. The challenge of providing better care for patients with chronic obstructive pulmonary disease: the poor relation of airway obstruction? Thorax 2000; 55 : $78-82$

8 Rennard SI. Combination bronchodilator therapy in COPD. Chest 1995; 107(suppl):1715-1755

9 Lightbody IM, Ingram CG, Legge JS, et al. Ipratropium bromide, salbutamol and prednisolone in bronchial asthma and chronic bronchitis. Br J Dis Chest 1978; 72:181-186

10 Casali L, Grassi C, Rampulla C, et al. Clinical pharmacology of a combination of bronchodilators. Int J Clin Pharmacol Biopharm 1979; 7:277-280

11 Brown IG, Chan CS, Kelly CA, et al. Assessment of the clinical usefulness of nebulised ipratropium bromide in patients with chronic airflow limitation. Thorax 1984; 39:272276

12 Gross NJ, Skorodin MS. Role of parasympathetic system in airway obstruction due to emphysema. N Engl J Med 1984; 311:421-425

13 Grassi V, Brunni B, Peccini F, et al. Acute and chronic comparative effects of a combination of fenoterol-ipratropium bromide and terbutaline in patients with chronic obstructive lung disease. Respiration 1986; 50(suppl 2):226-231

14 Wilson JD, Serby CW, Menjoge SS, et al. The efficacy and safety of combination bronchodilator therapy. Eur Respir Rev 1996; 6:286-289

15 Combivent Inhalation Aerosol Study Group. In chronic obstructive pulmonary disease, a combination of ipratropium and albuterol is more effective than either agent alone. Chest 1994; 105:1411-1419

16 Ikeda A, Nishimura K, Koyama H, et al. Bronchodilating effects of combined therapy with clinical dosages of ipratropium bromide and salbutamol for stable COPD: comparison with ipratropium bromide alone. Chest 1995; 107:401-405

17 van Noord JA, de Munck DRAJ, Bantje ThA, et al. Long-term treatment of chronic obstructive pulmonary disease with salmeterol and the additive effect of ipratropium. Eur Respir J 2000; 15:878-885

18 Pauwels RA. National and international guidelines for COPD. Chest 2000; 117(suppl):20S-22S

19 Bouros D, Bachlitzanakis N, Kottakis J, et al. Formoterol and beclomethasone versus higher dose beclomethasone as maintenance therapy in adult asthma. Eur Respir J 1999; 14:627632

20 Jones PW, Quirk FH, Baveystock CM, et al. A self-complete measure of health status for chronic airflow limitation: the St. George's Respiratory Questionnaire. Am Rev Respir Dis 1992; 145:1321-1327
21 US Food and Drug Administration, Department of Health and Human Services. ICH E9, International Conference on Harmonisation: guidance on statistical principles for clinical trials; availability. 63 Federal Register 179 (1998)

22 Grove A, Lipworth BJ, Reid RP, et al. Effects of regular salmeterol on lung function and exercise capacity in patients with chronic obstructive airways disease. Thorax 1996; 51: 689-693

23 Anderson GP. Formoterol: pharmacology, molecular basis of agonism, and mechanism of long duration of a highly potent and selective $\beta_{2}$-adrenoceptor agonist bronchodilator. Life Sci 1993; 52:2145-2160

24 Rebuck AS, Read J. Assessment and management of severe asthma. Am J Med 1971; 51:788-798

25 Abinsheganaden J, Sin Fai Lam KN, Lim TK. A profile of acute asthma patients presenting to the emergency room. Singapore Med J 1996; 37:252-254

26 Reddel H, Ware S, Marks G, et al. Differences between asthma exacerbations and poor asthma control. Lancet 1999; 353:364-369

27 Busse WW. Decreased granulocyte response to isoproterenol in asthma during upper respiratory infection. Am Rev Respir Dis 1977 ; 115:783-790

28 Buckner CK, Clayton DE, Ain-Shoka AA, et al. Parainfluenza 3 infection blocks the ability of a $\beta$ adrenoceptor agonist to inhibit antigen-induced contraction of guinea pig isolated airway smooth muscle. J Clin Invest 1981; 67:376-384

29 Bai TR, Mak JCW, Barnes PJ. A comparison of $\beta$-adrenergic receptors and in vitro relaxant responses to isoproterenol in asthmatic airway smooth muscle. Am J Respir Cell Mol Biol 1992; 6:647-651

30 Goldie RG, Spina D, Henry PJ, et al. In vitro responsiveness in human asthmatic bronchus to carbachol, histamine, $\beta$-adrenoceptor agonists and theophylline. Br J Clin Pharmacol 1986; 22:669-676

31 Cazzola M, Santangelo G, Piccolo A, et al. Effect of salmeterol and formoterol in patients with chronic obstructive pulmonary disease. Pulm Pharmacol 1994; 7:103-107

32 Van Noord JA, Smeets JJ, Raaijmakers JA, et al. Salmeterol versus formoterol in patients with moderately severe asthma: onset and duration of action. Eur Respir J 1996; 9:1684-1688

33 Sichletidis L, Kottakis J, Marcou S, et al. Bronchodilatory responses to formoterol, ipratropium and their combination in patients with stable COPD. Int J Clin Pract 1999; 53:185188

34 Dahl R, Greefhorst APM, Nowak D, et al. Comparison of the efficacy and safety of inhaled formoterol and ipratropium bromide in patients with COPD [abstract]. Am J Respir Crit Care Med 2000; 161(suppl):A489 